\title{
Amostras de ciclos autorregulatórios e sua inserção no processo de autoaprendizagem musical
}

\section{Examples of self-regulating cycles and their insertion in the process of musical self-learning}

\author{
iD) Antenor Ferreira Corrêa ${ }^{1}$ \\ Universidade de Brasília, Brasília,Brasil \\ antenorferreira@yahoo.com.br \\ (iD) Luciana Stadniki Morato Martins² \\ Universidade de Brasília, Brasília,Brasil \\ lu.smmartins@gmail.com
}

Resumo: Neste artigo são apresentados e exemplificados os conceitos da autorregulação e de ciclos autorregulatórios no aprendizado de um instrumento musical. O objetivo a ser atingido com a adoção da autorregulação está além da consecução da habilidade técnica para o desempenho de um instrumento musical, pois visa ao desenvolvimento metacognitivo do indivíduo. A proposição de ciclos autorregulatórios foi motivada, sobretudo, pelos escritos do psicólogo Barry Zimmerman (1998, 2000, 2002). Como resultado das considerações aqui empreendidas, apontamos a eficácia cognitiva do emprego da autorregulação, pois esse procedimento contribui para a autonomia do aluno que, dessa forma, se torna responsável por todas as etapas de seu processo de aprendizagem.

Palavras-chave: Ciclos autorregulatórios. Metacognição. Pedagogia da Performance Musical.

\footnotetext{
1 Professor Associado da Universidade de Brasília. Compositor, percussionista, arranjador e produtor musical. Possui pós-doutorado pela Universidade da Califórnia, Riverside. Pós-doutorado pela Universidade de Granada, Espanha. Doutor pela Univerdade de São Paulo, Brasil. Autor dos livros Music in an Intercultural Perspective (2016); A Mente Musical em uma Perspectiva Interdisciplinar (2015); Análise Musical Como Princípio Composicional (2014).

2 Professora da Escola de Música de Brasília; Flautista da Orquestra Sinfônica do Teatro Nacional Claudio Santoro
} 
Amostras de ciclos autorregulatórios e sua inserção....

Antenor Ferreira Correa • Luciana Stadniki Martins

Abstract: in this article we present and exemplify the concepts of self-regulation and self-regulating cycles in the teaching of musical instruments. The objective to be achieved with the adoption of self-regulation goes beyond the attainment of technical ability to perform a musical instrument, since it aims at the metacognitive development of the individual. The proposition of self-regulatory cycles was motivated mainly by the writings of the psychologist Barry Zimmerman (1998, 2000, 2002). As a result of the subjects here discussed, we point to the cognitive efficacy of the use of self-regulation, since this strategy contributes to the student's autonomy, which in this way becomesresponsible for all stages of his/ hers learning process.

Keywords:Self-regulatory cycles. Metacognition. Musical performance pedagogy.

Recebimento: 22/06/2019

Aceito em: 21/11/2019 


\section{Introdução}

A atividade docente direcionada ao ensino de um instrumento musical encontra-se, por vezes, entre Cila e Caríbdis. O progresso de um aluno resulta da dedicação que ele tem para com seu próprio aprendizado. Em outras palavras, o desenvolvimento do estudante depende da sua prática individual. Nesse sentido, muitos professores e muitas pedagogias acabam por cair no dilema "como" versus "quanto". Em vista disso, algumas abordagens didáticas decidem concentrar-se em como o aluno realiza sua prática cotidiana, ao passo que outras dedicam-se a instruir quanto tempo seria o ideal para a realização das diversas tarefas rotineiras que objetivam o domínio técnico de um instrumento musical.

Ambos os aspectos são, notadamente, fundamentais para o aprendizado e não pretendemos aqui advogar em favor de algum destes. Todavia, ao longo de nossa prática docente exercida em diversas instituições, para distintos públicos-alvo, e em conversas com vários colegas de profissão, observamos que a ênfase sobre "como" os professores querem que o aluno estude supera as recomendações sobre "quanto" tempo deve ser disponibilizado para cada tarefa. Em diálogos informais com os professores de instrumento, notamos que este aspecto é, na maior parte, negligenciado. Entendemos que, juntamente às prescrições e estratégias para a aquisição das habilidades técnicas para o domínio do instrumento, a otimização do tempo de estudo é fundamental para o bom rendimento, para a retenção de conteúdos e para a condução do estudante à sua autonomia.

Desse modo, percebemos a necessidade de orientar os estudantes para a organização autônoma e eficaz dos procedimentos que podem ser adotados em sua prática individual, com o objetivo de otimizar o tempo empreendido na realização das tarefas propostas. Para tanto, as definições claras das estratégias cognitivas direcionadas ao automonitoramento dessas atividades torna-se fundamental. O ponto de partida para a consecução desse objetivo está baseado na ideia de autorregulação. 
Amostras de ciclos autorregulatórios e sua inserção....

O conceito da autorregulação foi forjado a partir da Teoria Social Cognitiva (TSC) pelo psicólogo e pesquisador canadense Albert Bandura em 1986. A autorregulação, ou autocontrole comportamental, é um dos elementos essenciais para que o indivíduo viva em comunidade. De modo resumido, é possível indicar alguns aspectos intrínsecos ao conceito de autorregulação (BANDURA, 2017). A auto-observação, capacidade de não apenas se ver, mas principalmente de conseguir analisar e entender a si próprio. $O$ autojulgamento, que é a aptidão que o indivíduo tem de avaliar a si mesmo por meio de comparação com outras pessoas, com objetos ou consigo mesmo. Nesse sentido, uma pessoa pode julgar seu desempenho tendo por critério a performance de outras pessoas ou de uma máquina (ganhar uma partida de xadrez contra um computador ou sair vitorioso jogando videogame). Entretanto, a pessoa pode também ter a si mesma como modelo e meta a ser ultrapassada (vender mais do que vendeu no mês passado, tocar melhor do que tocou no último recital, correr o mesmo percurso em um tempo menor etc.). E a autorresposta (self-feedback), que é o mecanismo pelo qual a pessoa recompensa seu comportamento, positiva ou negativamente, em comparação a algum modelo escolhido como critério de aferição. Estes três aspectos, auto-observação, autojulgamento e autorresposta, estão na base da construção da autoestima, pois quando o indivíduo entende que obteve um desempenho melhor ou próximo do padrão adotado, sua autoestima aumenta. Do contrário, quando se sente aquém do modelo, sua autoestima tende a diminuir.

Nos parâmetros da TSC, Polydoro e Azzi (2008, p. 150) contextualizam os aspectos introdutórios da autorregulação proposta por Bandura, em que cada um é um ser capaz de exercer um papel ativo em sua própria história. Portanto, a autorregulação oferece, dentre as capacidades humanas, condições de monitoramento, avaliação e controle pelo indivíduo de seu próprio comportamento, em direção aos objetivos pessoais.

Entende-se como aprendizagem autorregulada ou a autorregulação do indivíduo, "a capacidade de autoensino, ou seja, ser 
Amostras de ciclos autorregulatórios e sua inserção....

Antenor Ferreira Correa • Luciana Stadniki Martins

capaz de preparar, facilitar e controlar a própria aprendizagem, proporcionar feedback e julgamento quanto aos resultados" (CAVALCANTI, 2010, p. 76). Este processo cognitivo apresenta conceituações igualmente exploradas por Paris e Paris (2001, p. 89), ao afirmarem que a aprendizagem autorregulada "enfatiza a autonomia e o controle por parte dos indivíduos que monitoram, dirigem e regulam as ações em direção às metas de aquisição de informação, expansão de conhecimento e auto aperfeiçoamento ${ }^{3 \prime}$.

Ao longo de nossa prática pedagógica como professores de instrumento, notamos que os alunos sentem-se responsáveis pelo próprio aprendizado a partir do momento que entendem as dimensões cognitivas do processo de autorregulação, em que o aluno, colaborando diretamente com o professor, define metas, elabora estratégias para atingi-las e cria ferramentas para se avaliar.

Em vista disso, intentamos aqui promover uma reflexão a partir do lugar do sujeito envolvido no seu próprio fazer musical, propondo abordar neste artigo a relação que cada indivíduo estabelece com o seu processo particular de aprendizado. $\mathrm{O}$ objetivo é incentivar o aluno à organização das ações para sua autoaprendizagem, desse modo sugerimos a apresentação de esquemas cíclicos dos processos da autorregulação como fonte de desenvolvimento sistematizado para que, a partir da ação metacognitiva, o aprendiz possa planejar, executar e avaliar seu progresso no desempenho das competências e habilidades necessárias para o seu aprimoramento musical.

Assim, a primeira etapa deste percurso é estabelecer um roteiro cíclico de estudos com diferentes possibilidades de metas, bem como as maneiras mais adequadas de realizá-las. Nesse processo, deve ficar evidente para o aluno não somente os objetivos elencados, mas também os aspectos para os quais ele deverá se atentar visando ao devido estabelecimento de uma prática autorregulada. Dentre esses aspectos estão o automonitoramento das estratégias de execução e de autoavaliação dos resultados alcançados.

3 Emphasizes autonomy and control by the individual who monitors, directs, and regulates actions toward goals of information acquisition, expanding expertise, and self-improvement (PARIS e PARIS, 2001, p. 89). 
Amostras de ciclos autorregulatórios e sua inserção....

Em seguida, deve-se explicitar a importância de o próprio aluno realizar a organização cíclica do seu tempo de prática, inserindo intervalos entre as seções de estudo, construindo e percebendo, dia a dia, a evolução do seu desempenho. Dessa maneira, espera-se que o aluno, ao encontro da aprendizagem autônoma, compreenda que, esgotadas as possibilidades de seus próprios recursos cognitivos para a resolução de dúvidas ou problemas de habilidades decorrentes das estratégias do estudo, é o momento de recorrer ao professor.

No campo da pedagogia da música, a promoção da prática cíclica dos processos autorregulatórios parte da indagação sobre como possibilitar aos alunos estratégias de autorregulação e, assim, contribuir para a operacionalização das questões de desempenho. A partir dessa interrogação, no decorrer desse texto são considerados alguns exemplos de esquemas cíclicos e suas respectivas especificidades em cada etapa do processo, de maneira a viabilizar escolhas e fomentar a autoadministração do estudo. O desenvolvimento metacognitivo é apresentado, paralelamente, como função mental da condução técnica e musical para que o processo ocorra de maneira eficiente.

O conceito de metacognição na aprendizagem torna-se um constructo para a fundamentação cognitiva da autorregulação na aprendizagem. Desse modo, desejamos vincular o campo metacognitivo ao processo da autorregulação na aprendizagem (SOARES, 2018) compreendendo que a metacognição atua no âmbito reflexivo, enquanto a autorregulação centra-se na ação prática.

Na década de 1970, Flavell (1979) explorou o termo metacognição a partir de três categorias principais: a pessoa, a tarefa e a estratégia, sendo que a categoria "pessoa" engloba os aspectos sobre a natureza de si mesmo como agente processador cognitivo. Segundo o autor, "o conhecimento metacognitivo consiste, principalmente, no conhecimento ou nas convicções sobre quais fatores ou variáveis agem e interagem de maneira a afetar o curso e o resultado dos empreendimentos cognitivos ${ }^{4 \prime \prime}$ (FLAVELL, 1979, p. 907).

4 Metacognitive knowledge consists primarily of knowledge or beliefs about what factors or variables act and interact in what ways to affect the course and outcome of cognitive enterprises (FLAVELL, 1979, p. 907). 
Assim, a expectativa é que a tomada de consciência do aluno sobre as estratégias de autorregulação, por meio de esquemas cíclicos e demais aspectos operacionais - como a elaboração de metas e táticas mais adequadas em contextos específicos de aprendizagem, bem como o controle de execução e a autoavaliação -, resultará no progresso do seu desempenho musical.

Sobre esse aspecto, Rosário (2014, p. 27), ao analisar diferentes considerações sobre a meta-análise e suas intervenções nos processos autorregulatórios na aprendizagem, percebeu que as dificuldades dos aprendizes para completar as tarefas de forma independente estavam associadas ao fato de o conhecimento metacognitivo compreender estratégias pouco robustas. A mudança desse quadro insatisfatório deveria considerar não apenas o treino dos componentes cognitivos, como a compreensão e elaboração das possibilidades de estratégias da aprendizagem, mas também a intervenção metacognitiva consciente, que, por sua vez, envolveria a tomada de decisões sobre os procedimentos, planejamentos, fatores motivacionais envolvidos e o feedback.

A importância de considerar o desenvolvimento da aprendizagem por meio da autorregulação justifica-se por possibilitar aos alunos situações em que possam refletir sobre seus próprios processos cognitivos a partir de um rol de atividades práticas. O que envolve competências sistêmicas ligadas ao planejamento dos ciclos autorregulatórios como método de otimização de seu tempo de estudo. Assim, o aluno é incentivado a refletir sobre quando, onde e de que maneira aplicará as estratégias de aprendizagem.

A defesa pela busca do desenvolvimento discente mediado pelas capacitações metacognitivas, a partir da orientação do professor, é assim apresentada por Simão:

Face à não utilização, de forma autônoma, das estratégias de aprendizagem, começamos por ensinar a aprendê-las de forma sistemática. Procuramos desenvolver as capacidades de pensamento dos alunos potencializando as competências metacognitivas, pois não podemos dissociar o ensino de 
Amostras de ciclos autorregulatórios e sua inserção....

Antenor Ferreira Correa • Luciana Stadniki Martins

estratégias e a atividade metacognitiva quando pretendemos aspirar a conseguir mudanças que sejam transferidas e realizadas de forma autônoma pelos alunos (SIMÃO, 2002, p. 285).

Dessa maneira, inserir no cotidiano educacional atividades visando ao desenvolvimento metacognitivo consequentemente fortalecerá a consciência da autorregulação que, por seu turno, contribui para a autonomia do aluno em relação ao seu aprendizado.

\section{Os ciclos autorregulatórios e suas implicações práticas no ensino e aprendizagem na área da música}

Como discorrido na Introdução, a ideia de autorregulação está contemplada na Teoria Social Cognitiva e tem sido atualizada em alguns ramos da psicologia atual, principalmente em pesquisas da psicologia da educação (vide adiante). A autorregulação consiste na capacidade de reflexão do indivíduo sobre suas ações e comportamentos de maneira voluntária e consciente, com a possibilidade cíclica de recuperação, julgamento e adaptação dos resultados de sua própria atuação. Consequentemente, ela envolve métodos e processos direcionados para que os alunos se tornem autorreguladores de seu processo de aprendizagem. Por conta disso, algumas propostas de autorregulação instrucional levaram os estudiosos a desenvolver modelos cíclicos divididos por fases de desenvolvimento. Em geral, tais ciclos envolvem fundamentalmente as metas de monitoramento, autoavaliação e o uso de estratégias, sendo que, quanto mais capazes os alunos se julgarem, mais desafiadores serão os objetivos que eles adotam (ZIMMERMAN, 2000, p.87).

Sob a ótica da teoria sociocognitiva, o sistema proposto por Bandura (1978, p. 348) expõe, basicamente, as funções da autopercepção (auto-observação) do monitoramento das ações e a autoavaliação como pilares do processo. Essas ações cíclicas autorreguladas, ao longo das últimas décadas, têm sido objeto de 
Amostras de ciclos autorregulatórios e sua inserção....

Antenor Ferreira Correa • Luciana Stadniki Martins

estudo de diversas pesquisas, cujos resultados ofereceram um conteúdo propositivo muito sólido, sobretudo voltado para a edificação de estratégias de ensino e aprendizagem (ZIMMERMAN, 1998, 2000, 2002; WINNE; HADWIN, 1998; SIMÃO, 2002; SCHUNK; ZIMMERMAN, 2006; ROSÁRIO, 2014) e de propostas de ciclos autorregulatórios direcionadas para o campo da música (GALVÃO, 2006; MCPHERSON; ZIMMERMAN, 2011; ZORZAL, 2014).

De maneira abrangente, para alunos autorregulados o processo de aprendizagem apresenta-se como uma atividade proativa que exige encadeamentos metacognitivos, motivacionais e comportamentais com definições de objetivos personalizados. Esse processo ocorre associado ao automonitoramento do pensamento estratégico. Para esses estudantes, agentes controladores de seu próprio aprendizado, a autorregulação é um processo autodiretivo por meio do qual eles transformam suas habilidades mentais em habilidades acadêmicas. "A aprendizagem é vista como uma atividade que os alunos realizam por si mesmos de uma maneira proativa, e não como um evento encoberto que acontece em reação ao ensino. A auto-regulação refere-se a pensamentos, sentimentos e comportamentos auto-gerados, orientados para a consecução de objetivos" ${ }^{\text {5" }}$ (ZIMMERMAN, 2002, p. 65).

A Figura 1 (abaixo) apresenta um modelo cíclico da autorregulação formulado por Zimmerman e Campillo (2003), sintetizando a proposta dos autores de maneira contínua em três etapas. A análise, especificamente desse ciclo, abarca os teóricos Albert Bandura e Barry Zimmerman, que fundamentam os sistemas cíclicos da aprendizagem autorregulada e suas visões das fases e subprocessos que o compõem.

Baseada na autorregulação acadêmica, Zimmerman (1998, p. 3; ZIMMERMAN et al. 2002, p. 67) apresenta uma estrutura cíclica do processo dividida em três fases. Como mostrado na Figura 1, a primeira fase consiste da premeditação (Forethought Phase), referindo-se aos processos e crenças da autoeficácia que ocorrem

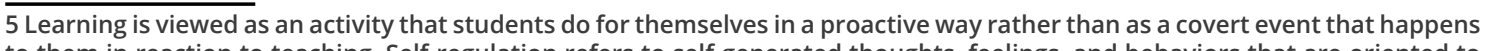
to them in reaction to teaching. Self-regulation refers to self-generated thoughts, feelings, and behaviors that are oriented to attaining goals (ZIMMERMAN, 2002, p. 65). 
Amostras de ciclos autorregulatórios e sua inserção....

Antenor Ferreira Correa • Luciana Stadniki Martins

antes da aprendizagem e precedem os esforços para aprender e preparar o terreno para o aprendizado. Para Bandura (2008, p. 159), Zimmerman aponta nessa fase as variáveis antecedentes, que envolvem um resultado esperado, por meio do planejamento de estratégias e metas de realização, das crenças de autoeficácia e, consequentemente, do interesse intrínseco.

\section{Figura 1: Fases e subprocessos da autorregulação propostos por Zimmerman e Campillo (2003).}
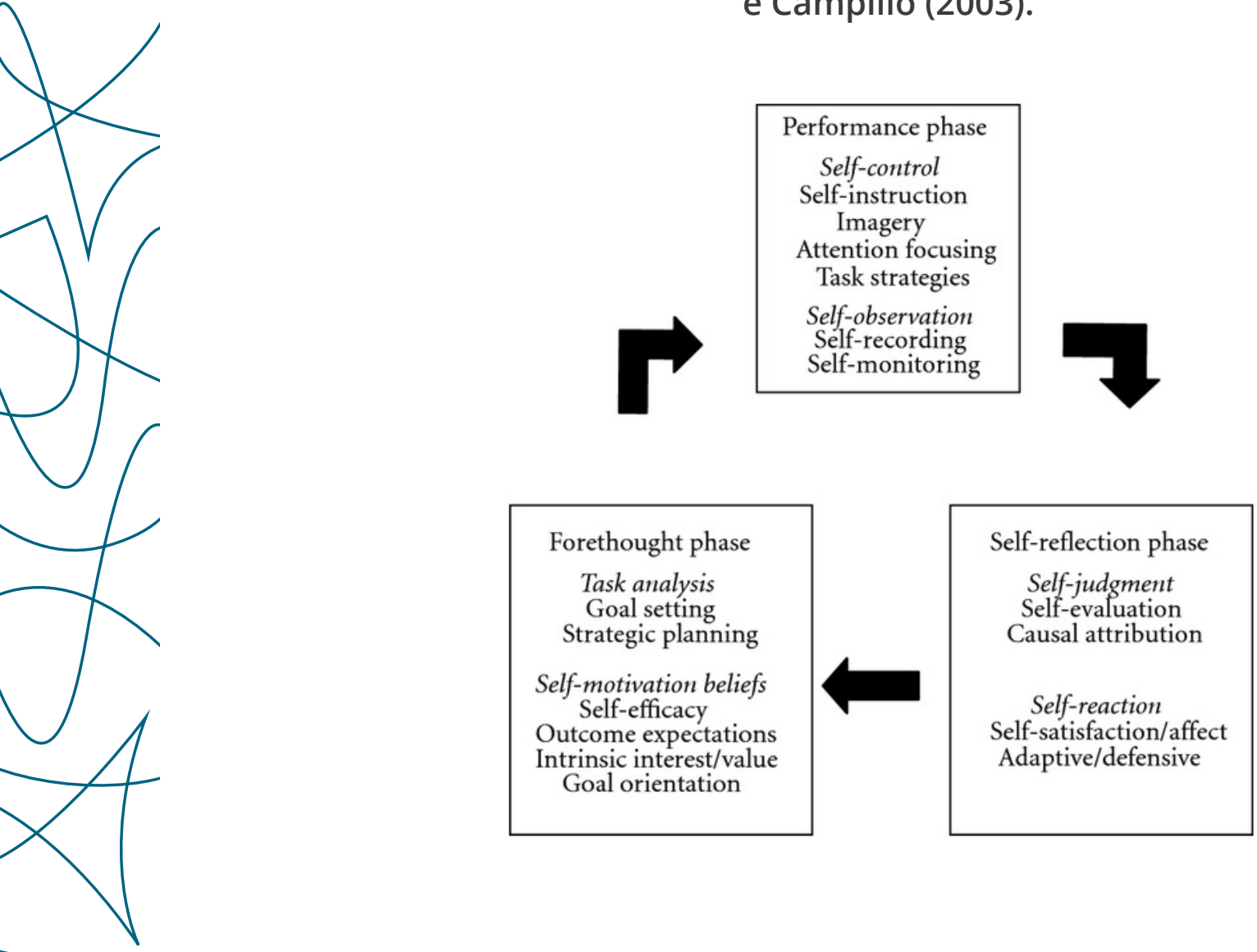

Na segunda fase dá-se o controle de desempenho (Performance Phase), que diz respeito aos processos gerados na implementação comportamental e envolve procedimentos que ocorrem durante os esforços de aprendizagem. Esses procedimentos afetam a concentração e o próprio desempenho (ZIMMERMANN, 1998, p. 3). Bandura avalia essa segunda fase como o controle de desempenho e da motivação ativos durante todo esse processo de 
aprendizagem da autorregulação, que compreende, nessa etapa cíclica, a concentração, a autoinstrução e o automonitoramento.

A última fase do ciclo apresentado por Zimmerman corresponde à autorreflexão (Self-Reflection Phase), que acontece após cada esforço da aprendizagem e influencia as reações dos aprendizes à experiência realizada. As autorreflexões influenciam a premeditação em relação aos esforços subsequentes da aprendizagem, completando assim o ciclo da autorregulação. Nessa fase existem duas principais classes de previsão: a análise de tarefas e a automotivação. Segundo Bandura, na fase da autorreflexão, que ocorre após o processo de aprendizagem, ativam-se atribuições como a autoavaliação, as autorreações e a adaptação ao início do próximo ciclo.

No campo da psicologia da aprendizagem autorregulada, o modelo cíclico apresentado por Rosário (2014, p. 39) tem por base o modelo de Zimmerman e mostra a caracterização intrinsecamente cíclica e interdependente dos processos de autorregulação que originam um modelo cíclico composto também por intrafases, denominado pelo autor de PLEA (planejamento, execução e avaliação), vide Figura 2 a seguir.

Figura 2: Modelo PLEA proposto por Pedro Rosário (2014, p. 39).

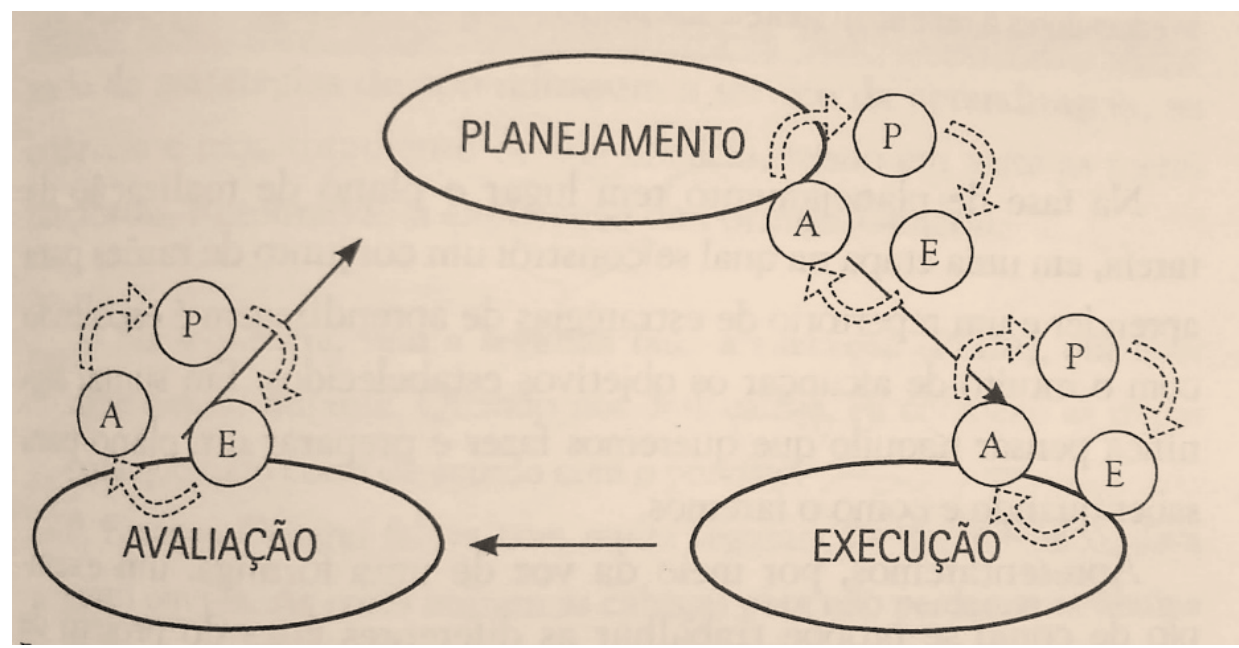


A partir de duas lógicas cíclicas, uma com o ciclo completo e a outra por sobreposição de cada fase em seu próprio movimento cíclico, a proposta permite uma outra visão operacional do ciclo autorregulatório: com reforço a partir da lógica da organização das fases do planejamento, da execução e da avaliação e com proposições de uma dinâmica cíclica em cada uma das fases. Esse processo permite ao aluno a revisão mais detalhada de cada etapa cíclica, como forma de garantir uma melhor qualidade e avaliação do processo.

Outro modelo igualmente referenciado entre os estudiosos dos ciclos da aprendizagem autorregulada é o de Winne e Hadwin (1998, p. 282). A escolha da análise desse modelo se justifica pela possibilidade de extensão e desmembramento do modelo triádico para a composição cíclica, em números maiores de fases. Segundo esses pesquisadores, as estratégias autorregulatórias que antecedem a elaboração das metas envolvem a definição dos recursos a serem utilizados, bem como os espaços e objetivos preliminares e, assim, esse modelo apresenta-se dividido em 4 estágios:

Estágio 1: Definição das atividades. Nessa fase, o aprendiz desenvolve uma percepção dos recursos das atividades propostas, mapeando-a como um "espaço de estudo". Um importante elemento desse estágio é a possibilidade do aluno antever as metas a serem cumpridas. Por exemplo, separar o material a ser estudado. Nesse estágio, o aluno poderá definir o repertório a ser estudado de acordo com o seu nível técnico. Geralmente, os métodos de estudo possuem uma progressividade técnica e musical que pode servir de auxílio para a construção desse estágio. Os exercícios que tratam das habilidades técnicas em escalas e arpejos poderão ajudar e também devem fazer parte desse levantamento.

Estágio 2: Definição das metas e planejamento. No estágio 2 do estudo, o aluno pode redefinir as metas que foram programadas no estágio 1 , com base em seus padrões pessoais, diferindo das anteriores. De acordo com a disponibilidade de tempo semanal para o cumprimento das atividades definidas no estágio 1 , essas metas poderão sofrer adaptações no segundo estágio. As 
Amostras de ciclos autorregulatórios e sua inserção....

adaptações fazem parte do processo e garantem uma melhor organização das metas a serem alcançadas.

Estágio 3: Deliberar táticas e estratégias de estudo. O estágio 3 envolve a realização efetiva das táticas de estudo e estratégias planejadas durante o estágio 2. Entretanto, na medida em que essas operações são aplicadas, as avaliações (feedback) são geradas internamente. Nesse estágio, diferentes estratégias podem ser utilizadas, por exemplo:

1. Isole os trechos em que tem dificuldade;

2. Repita cada trecho em velocidades progressivas;

3. Contextualize o trecho: execute a frase anterior, seguida pelo trecho em dificuldade e a frase posterior ao trecho;

4. "Desconstrua" o trecho: executando de trás para frente. Comece executando a última nota e ao longo das repetições vá acrescentando cada nota, em sequência: a penúltima com a última, a antepenúltima com a penúltima e a última e assim por diante, até reconstruir o trecho isolado;

5. Grave, escute e analise sua própria execução;

6. Compare a sua execução com a do professor.

Estágio 4: Estudo metacognitivo adaptado. No estágio 4, o aluno inspeciona as estratégias criadas nos estágios anteriores com o monitoramento da tática utilizada em todo o processo. Depois do feedback é importante observar se as estratégias foram eficazes. Criar condições de apresentação é uma boa oportunidade de colocar em prática a eficácia do processo.

Esses estágios sugeridos por Winne e Hadwin indicam procedimentos preliminares à prática no instrumento. Trata-se de um planejamento que mostrará ao estudante a importância de suas escolhas prévias, pois cada escolha implicará particularidades a serem vencidas para a conquista de um bom desempenho técnico.

Para as adaptações das propostas cíclicas no campo da autorregulação musical, McPherson e Zimmerman, em relação ao controle do desempenho autorregulado, propõem basicamente dois 
Amostras de ciclos autorregulatórios e sua inserção....

Antenor Ferreira Correa • Luciana Stadniki Martins

processos gerais que capacitam os alunos a otimizar sua própria atuação, a saber,

o primeiro processo de autocontrole ajuda o músico a otimizar seus esforços através de meios como autoinstruções (explicitamente ou secretamente descrevendo como executar uma tarefa), imagens, foco da atenção e estratégias de atividades. O segundo processo de desempenho envolve a autoobservação, que ajuda a informar o aluno sobre a sua evolução ou a falta do seu progresso (2011, p. 160).

As reflexões propostas por McPherson e Zimmerman (2011, p. 159) partem de pensamentos e crenças pessoais para o engajamento de esforços em uma tarefa, o que pode definir e influenciar o aprendizado subsequente. Esse controle evolucionário afeta diretamente processos que ocorrem durante o aprendizado, como a concentração e o desempenho da performance. Já a autorreflexão, após o aprendizado, induz reações no aluno para a premeditação de respostas subsequentes à experiência e elaboração de futuros esforços da aprendizagem. Para os autores, quando um aluno aprende um instrumento musical, o propósito de definir metas a curto e longo prazo permite que se estabeleçam padrões pessoais de desempenho, interferindo diretamente no processo motivacional. Assim, alunos que estabelecem metas claras de desempenho para si, principalmente diante de uma dificuldade, conseguem ser mais persistentes no cumprimento das instruções, concentram melhor seus esforços e são mais propensos a obter prazer e confiança em suas habilidades.

Em relação às estratégias do ciclo de autorregulação no aprendizado musical, Galvão (2006, p. 171) propõe um modelo mais seccionado, constituído de "organização, transformação de informação, sequenciamento, busca de informação, ensaio e estratégias mnemônicas".

Durante o processo cíclico de autorregulação, a passagem do estágio cognitivo inicial leva à organização de procedimentos por 
Amostras de ciclos autorregulatórios e sua inserção....

Antenor Ferreira Correa • Luciana Stadniki Martins

fragmentos e regras que, especificamente na aquisição da habilidade técnica musical, para Galvão (2015, p. 170) "determinam sequências, envolvendo a prática de parte de uma habilidade antes de tentá-la de um modo completo para atingir o domínio final da tarefa". O autor considera os experimentos de Hallam (1997) ao afirmar que, com base na estrutura metacognitiva, o processo de aprendizagem multifacetado, em sua prática efetiva por músicos profissionais, tende a ser concretizado por uma preocupação em dominar fragmentos significativos para o texto musical, de modo a produzir resultados rápidos e eficientes (apud Galvão, 2015, p. 181).

Ainda como proposta de ciclo de autorregulação voltado para a aprendizagem musical e instrumental, Zorzal $(2015$, p. 97) apresenta um processo detalhado da prática efetiva, porém com um nível de performance já obtido. Segundo o autor, os fatores iniciais são exemplificados e sugerem uma interação mútua entre o nível de performance, a motivação (intrínseca e extrínseca), o ambiente de estudo, a qualidade da instrução recebida e a qualidade final de uma performance anterior, vide Figura 3.

Figura 3: Processo detalhado da prática efetiva proposto por Zorzal (2015, p. 98).

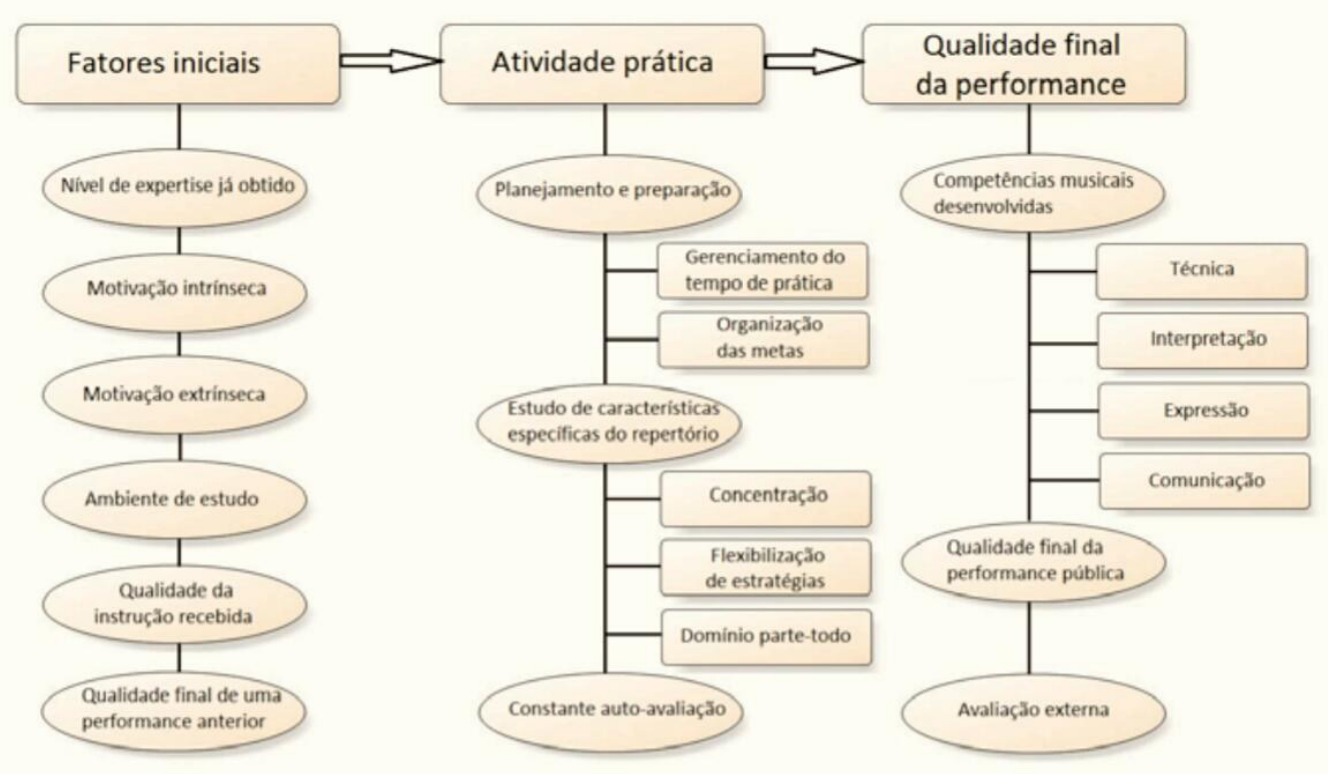


Duas importantes fundamentações atreladas ao processo exposto por Zorzal $(2015$, p. 98) se destacam para a prática do autoensino. A primeira é que a efetividade da prática exige, de quem a pratica, uma postura reflexiva que proporciona, ao longo do tempo, a capacidade da reflexão na ação. A segunda fundamentação é a possibilidade de estipular metas que sejam exequíveis em curtos prazos de tempo.

Uma análise geral dos modelos autorregulados apresentados aponta que o indivíduo pode refletir e intervir no ambiente externo a partir da identificação de seu próprio comportamento associada à qualidade de seu desempenho. Em consequência, as ações pessoais (escolhas do ambiente de estudo, material selecionado, metas para o cumprimento de tarefas, estratégias de execução, distribuição do tempo disponibilizado) e informações selecionadas (escolhas de exercícios e métodos específicos, referências de modelos profissionais como fonte de interpretação musical da execução das obras) são avaliadas no processo de julgamento, com a atribuição de valores das atividades, dos padrões pessoais e das normas sociais. A partir desse monitoramento e de reflexões pessoais, as subfunções que surgem no ciclo a partir da autorreação representam transformações dirigidas pelo próprio indivíduo com base em resultados autoadministrados. Dessa forma, uma retroalimentação (feedback) do processo se apresenta para o início de um novo fluxo de auto-observação.

Os modelos apresentados nesse artigo foram apurados pelas fundamentações cíclicas da autorregulação, a exemplo da teoria social cognitiva, e sofreram suas extensões no campo da educação musical. De forma sucinta, as fases cíclicas para o processo autorregulatório na aprendizagem são apontadas da seguinte maneira por Zimmerman:

O objetivo de cada estratégia é melhorar a autorregulação do aluno, [...] estratégias de organização e transformação, reaprendizado e memorização, estabelecimento de metas e planejamento focados na otimização da regulamentação pessoal. 
Estratégias como autoavaliação e autoconsequências foram elaboradas para melhorar o funcionamento comportamental (1998, p. 7).

Por fim, na literatura da aprendizagem cíclica autorregulada, e adaptando os modelos ao campo do ensino da música com referências aos estágios do autoestudo, é consensual que os pilares do processo da autorregulação - como a organização, a motivação, a qualidade das metas de planejamento, o monitoramento e a adequação (feedback) - tornem-se importantes elementos cíclicos na dimensão da metacognição autorregulatória da performance instrumental. Na perspectiva de que os alunos adquiram as ferramentas necessárias para assumir o controle de sua aprendizagem, de acordo com Madeira (2014, p. 7), é “necessário que [estes] aprendam a monitorar as suas próprias estratégias, comportamentos e recursos cognitivos, ajustando-os constantemente às alterações das condições que ocorrem ao longo da aprendizagem".

\section{Exemplo prático da utilização cíclica da aprendizagem autorregulada}

Nossa experiência no ensino de instrumentos musicais permite fundamentar a percepção de que os alunos sentem-se responsáveis por sua própria aprendizagem quando são incluídos no processo pedagógico e motivados a pensar sobre o repertório que gostariam de tocar, assim como realizar uma distribuição eficaz de seu tempo de estudo, identificar situações problemáticas entre outros. Na dimensão da autorregulação voltada para o campo da música, diferentes estratégias são identificadas como ações cíclicas mais comuns utilizadas pelos alunos que praticam o estudo de um instrumento musical, elas incluem a observação por meio da escuta musical com referências de músicos profissionais e dos professores, além das diferentes maneiras de "como" estudar e a 
Amostras de ciclos autorregulatórios e sua inserção....

preocupação com o melhor aproveitamento do tempo disponibilizado para o cumprimento das metas.

Alguns autores compartilham estratégias da prática dos aspectos técnico-interpretativos das obras, incorporando-as à execução instrumental e automatizando os movimentos através, por exemplo, da repetição, que se apresenta como um importante mecanismo para o desenvolvimento e aprimoramento das habilidades musicais (GUIMARÃES, 2011; ADDESSI, 2015; SLOBODA, 2012). Segundo Freire, o processo de imitação consiste na repetição de determinada informação e é uma ferramenta fundamental para o processo de aprendizagem musical. Afinal, "a imitação operacional pode ser trabalhada a partir da repetição de pequenos grupos de três ou quatro notas, que precisam ser imitados imediatamente, para que as informações sejam mantidas ou ensaiadas mentalmente" $(2015$, p. 249).

A estratégia da repetição sequencial leva ao aprimoramento cinestésico (habilidade motora), como ação muscular conscientemente desenvolvida no processo de aprendizagem do instrumento (GALVÃO, 2015; CERQUEIRA, 2009; KAPLAN, 2008). Para Galvão (2015, p. 170), "o estudo individual é a organização da habilidade passo a passo. Trata-se do processo por meio do qual objetivos de aprendizagem são alcançados". Assim, a prática do estudo individual com a identificação de passos da ação mecânica sequencial é um componente essencial para a melhoria das habilidades técnicas musicais. Kaplan (2008, p. 36) defende que a execução de movimentos curtos e corretamente "calibrados" se tornam automatizados com a prática consciente.

Dessa maneira, a prática da repetição fragmentada, além de solucionar as dificuldades de uma determinada passagem, sedimenta movimentos que podem ser aplicados em diferentes contextos musicais. "Podemos perceber que quanto mais exercemos determinada atividade musical, mais conhecimentos acumulamos não somente sobre a atividade em si, mas também sobre atividades similares que venhamos a ter contato futuro" (CORRÊA, 2017, p. 66). De fato, alguns autores destacam que a prática persistente de 
Amostras de ciclos autorregulatórios e sua inserção....

uma habilidade a torna mais e mais "independente" de contextos específicos (SLOBODA, 2012, p. 44), possibilitando que informações importantes sejam armazenadas na memória de longa duração e acessadas pela memória de curto prazo. Desse modo, as informações armazenadas serão recuperadas em distintas atividades de percepção musical, pois serão reconhecidas como correspondentes àquelas já adquiridas.

Em relação às estratégias de organização do tempo de estudo, defendemos que a prática distribuída é melhor que a prática concentrada, o que significa estendê-la por vários períodos separados em vez de concentrá-la em uma única sessão. Por exemplo, com sete horas disponibilizadas na semana para se dedicar a uma prática específica, talvez seja melhor distribuir o horário em uma hora por dia, em vez de sete horas em um único dia. Diversos autores compartilham desse entendimento, Sloboda (2012) é um deles.

Criar um roteiro de estudo com definições e objetivos a serem alcançados a partir da escolha do repertório específico para o nível técnico do aluno torna o desenvolvimento da performance musical organizado e gradativo, com etapas progressivas das destrezas cinestésicas e da memória motora, bem como da maturidade da interpretação musical fraseológica a partir das contextualizações de estilos e análises funcionais e harmônicas da obra musical estudada.

Um exemplo prático é apresentado na Figura 4 (abaixo). O estudo escolhido é o de n. 7, do compositor e flautista italiano Ernesto Köhler (1849-1907), que faz parte de uma coletânea de 15 estudos fáceis para flauta, editado pela Ricordi ("15 Studi Facili per il Flauto", opus 33, vol.1).

Com base na estrutura cíclica, as fases podem ser definidas em três momentos do estudo, a saber: 
Parte 1: O levantamento das metas e as estratégias de estudo.

a. O aluno faz uma leitura visual da partitura, com coleta de informações sobre o andamento (Allegretto, com semínima a 120 bpm), a tonalidade (Ré menor) ou a armadura de clave, a unidade de tempo (semínima), além da análise estrutural dos temas musicais e das possíveis sessões como a exposição (compassos n. 1 ao n. 40), o desenvolvimento (compassos n. 41 ao n. 66), a reexposição (compassos n. 67 ao n. 81) e a coda (compassos n. 82 ao n. 92);

b. A escuta de referências com observação ativa e atenta, por meio de instrumentistas profissionais ou pelo professor, proporcionará um panorama geral da execução da obra e servirá também como exemplo de possibilidade interpretativa;

c. A execução da obra estudada poderá ser realizada em andamento mais lento do que a exigida pelo compositor e dividida por sessões, frases ou trechos cada vez menores, que exijam aperfeiçoamento técnico específico;

d. A repetição fracionada pode acontecer como uma montagem metaforicamente chamada de "colcha de retalhos" ou técnica em patchwok. O trecho em dificuldade é executado juntando a frase anterior com a posterior. Para o exemplo acima, supondo que a dificuldade se encontra no compasso n. 18, esse compasso é isolado e executado repetidas vezes. Depois disso, introduz-se o compasso anterior, n. 17, e executa-se o trecho entre os compassos n. 17 e 18. Na sequência o compasso posterior, n. 19, é introduzido e executam-se os compassos n. 18 e n. 19. Por fim, executa-se todo o trecho compreendido entre os compassos n. 17 e n. 19, montando-se, assim, os "retalhos" da colcha em todo o seu contexto musical. 
Amostras de ciclos autorregulatórios e sua inserção....

Antenor Ferreira Correa • Luciana Stadniki Martins

Figura 4: Estudo n. 7, opus 33 de Ernesto Köhler.

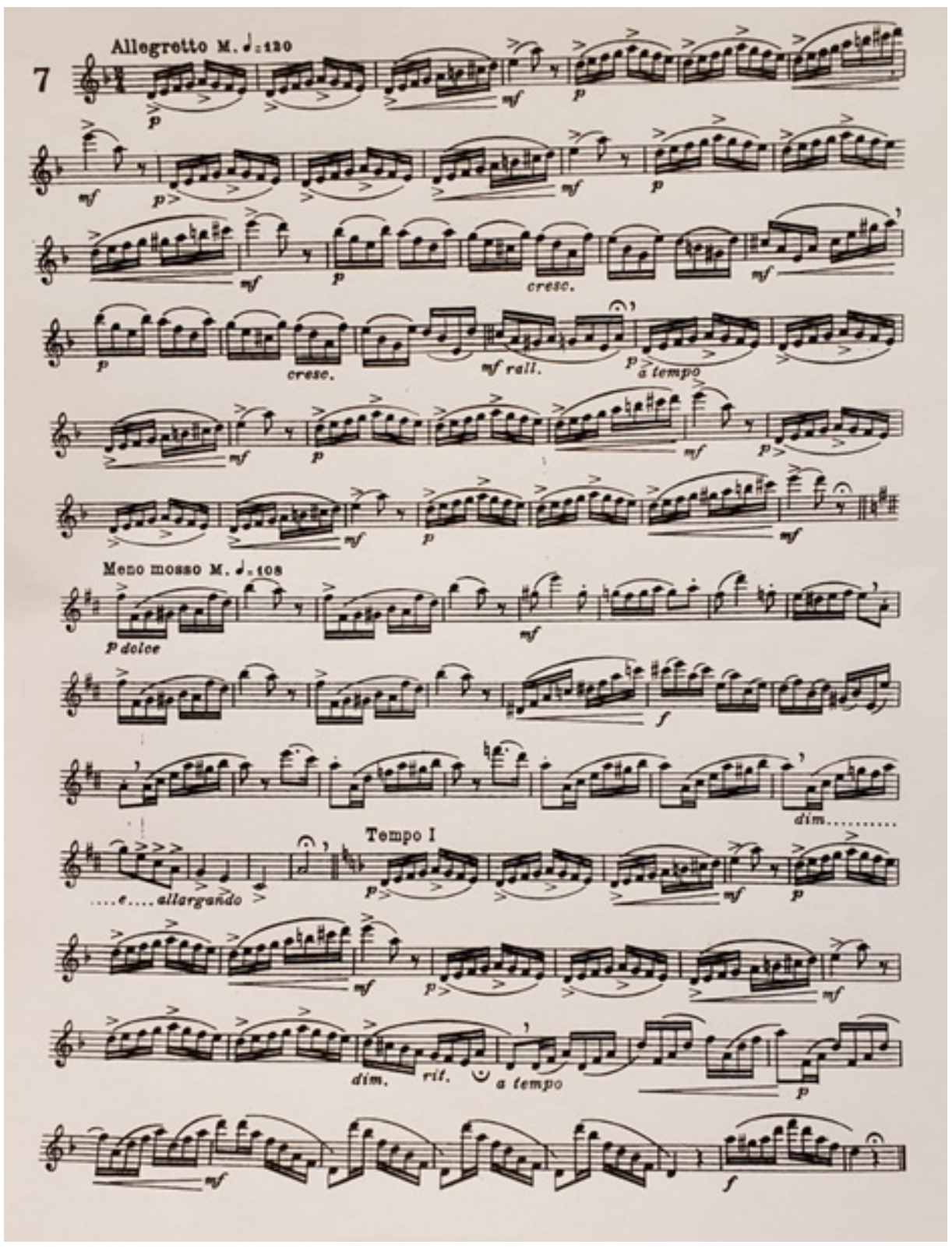

Parte 2: O monitoramento da execução.

A segunda fase cíclica envolve a análise das estratégias utilizadas para a execução do estudo, com intuito de avaliar sua eficácia. As metas e as estratégias escolhidas são analisadas nessa fase, assim como a distribuição do tempo de estudo. 
Amostras de ciclos autorregulatórios e sua inserção....

Antenor Ferreira Correa • Luciana Stadniki Martins

O aluno deve gravar sua própria execução e ouvi-la com o acompanhamento da partitura ou com recursos eletrônicos, como o metrônomo, a fim de monitorar sua própria execução.

\section{Parte 3: O feedback.}

Caso os resultados obtidos com as estratégias de estudo escolhidas sejam considerados insatisfatórios pelo aluno ou pela análise do professor/orientador, a próxima fase deverá fomentar mudanças na formulação do tempo de estudo e das técnicas utilizadas para o desenvolvimento da performance musical.

\section{Considerações finais}

Com base nos modelos cíclicos apresentados nesse texto, de maneira geral, é possível sintetizar que, para a autorregulação da aprendizagem sob o aspecto de sua periodicidade, a prática de estudo individual deve partir de uma organização da habilidade passo a passo para que os objetivos da aprendizagem sejam alcançados. Dessa maneira, o estudo voltado para a aquisição de uma habilidade envolve a identificação de estágios cognitivos iniciais que levam a uma ação sequencial. Tais ações poderão ser fragmentadas como parte da prática de uma determinada habilidade, antes de aplicá-la de modo completo.

De maneira geral, os ciclos aqui expostos apresentam um funcionamento em fases que se complementam e mantêm um movimento contínuo em busca de aperfeiçoamentos do desempenho. Para a aprendizagem musical, as adaptações apresentam uma fase prévia à ação musical e se estabelecem com a análise das tarefas, a criação de objetivos e o planejamento de estratégias, alimentando-se da automotivação e da autoeficácia apoiadas nas expectativas de alcance dos objetivos finais esperados. A segunda fase envolve a prática da performance e se constitui da implementação do plano estratégico da fase anterior, com a aplicação do 
autocontrole do plano estratégico e da autoinstrução e com foco na atenção e mobilização de estratégias estabelecidas. Por fim, a última etapa cíclica, porém contínua ao processo, finaliza o processamento das informações registradas durante a performance musical com vista à autorreflexão e à autoavaliação do resultado, da atividade e do esforço despendido. As reações dessa fase incluem a autossatisfação e a postura adaptativa ou defensiva perante o resultado obtido. Essa fase determina a readaptação de todo o processo, incluindo as estratégias e os métodos, o reajuste de objetivos e esforços, bem como a redefinição da valorização, persistência e motivação.

Finalmente, aliadas a esse processo, as instruções apropriadas do professor, em seu papel de agente modelador, juntamente às orientações cíclicas contextualizadas, têm como objetivo o desenvolvimento autônomo do aluno a partir da metacognição como estratégia de evolução e sucesso da proposta cíclica autorregulatória para o aprendizado musical. A partir do conhecimento que o aprendiz tem sobre si mesmo e sobre os mecanismos da aprendizagem, as fases e os processos cíclicos da autorregulação, espera-se que o aluno possa controlar quando e como utilizar estratégias e planejamentos de estudo, avaliar a eficácia dos procedimentos escolhidos, monitorar o tempo e o ambiente de estudo, dentre outros aspectos apontados, e assim assumir as diretrizes que poderão alterar o seu desempenho em busca da satisfação pessoal em seu fazer musical.

Espera-se que a exibição e as análises dos exemplos de ações cíclicas de desempenho apresentadas ao longo desse trabalho sirvam como fonte proveitosa para a construção autônoma do planejamento de estudo e que provoquem experiências significativas no sujeito em busca da melhoria da sua aprendizagem e do seu desenvolvimento musical. 
Amostras de ciclos autorregulatórios e sua inserção.... Antenor Ferreira Correa • Luciana Stadniki Martins

\section{Referências}

ADDESSI, ANNA RITA. O PARADIGMA DA INTERAÇÃO REFLEXIVA NO CAMPO DA APRENDIZAGEM MEDIADA POR TECNOLOGIAS: ARCABOUÇO TEÓRICO E ALGUNS RESULTAdoS empíricos COM A PLATAFORMa MIROR. IN: CORRÊA, ANTENOR Ferreira. A MENTE MUSICAL EM UMA PERSPECTIVA INTERDISCIPLINAR. BRASÍLIA: UNIVERSIDADE de Brasília, 2015. p.191-233. Tradução: Rosane Cardoso de Araújo.

Bandura, Albert. The self-system in reciprocal determinism. American Psychologist, Stanford, v. 33, N. 4, P. 344-358, aBr. 1978.

BANDURA, Albert. Social foundations of thought and action: A social cognitive theory. Englewood Cliffs, NJ: Prentice-Hall, 1986.

Bandura, Albert; AZZI, Roberta Gurgel; POlydoro, Soely. Teoria social cognitiva: conceitos básicos.Porto Alegre: Artmed, 2008. Tradução DO INGLÊS CAP. 1 AO 5: Ronaldo Costa.

BANDURA, Albert. ReConstrução do “livre arbítrio" a PARTIR dA PerSPECtiva aGÊNTICA DA TEORIA SOCIAL COGNITIVA. In: BANDURA, Albert; AZZI, RoberTA Gurgel. Teoria social cognitiva: diversos enfoques. Campinas: Mercado de Letras, 2017. Cap. 4. P. 129-200. Tradução: Ana Cecílla de Medeiros MACIEL.

CAVALCANTI, CÉlIA R. P. Um ESTUdo SOBRe a AUtORREGULAÇÃo da PRÁtICA instrumental de músicos instrumentistas. Música em Perspectiva: Revista do Programa de Pós-Graduação em Música da UFPR, Curitiba, v. 3, N. 2, P.74-86, OUT. 2010. DISPONÍVEL EM: DOI: HTTP://DX.DOI.ORG/10.5380/MP. V3I2.21983. ACESSO EM: 30 AGO. 2018.

Cerqueira, Daniel Lemos. Proposta para um modelo de ensino e aprendiZagem da performance musical. Opus, GoiÂnia, v. 15, N. 2, P. 105-124, dez. 2009.

Cerqueira, Daniel Lemos; OliVeira, Cristiano Braga de. Transmissão DE CONHECIMENTOS NO ENSINO DA PERFORMANCE MUSICAL. IN: ZORZAL, RICIERI Carlini; tourinho, Cristina (Org.). Aspectos práticos e teóricos para o ensino e aprendizagem da Performance musical. São Luís: EdufMA, 2014. CAP. 5. P. 170-219. 
Amostras de ciclos autorregulatórios e sua inserção....

Antenor Ferreira Correa • Luciana Stadniki Martins

CORrÊA, Antenor Ferreira. Por uma epistemologia da composição musiCal. Percepta: Revista de Cognição Musical, Curitiba, v. 4, N. 2, P. 57-69, 2017.

FlaVell, John H. Metacognition and Cognitive Monitoring: A New Area of Cognitive-Developmental Inquiry. American Psychologist, Stanford, v. 34, N. 10, P. 906-911, оUT. 1979.

FREIRE, Ricardo Dourado. ARTICULAÇões ENTRE IMITAÇÃo E MEMÓRIA NOS PRoCessos de percepção musical. In: CORRÊA, Antenor Ferreira (OrG.). A MENTE MUSICAL EM UMA PERSPECTIVA INTERDISCIPLINAR. BRASÍLIA: UNIVERSIDADE de Brasília, 2015. P. 235-254.

GAlvão, Afonso. Cognição, emoção e expertise musical. Psicologia: Teoria e Pesquisa, Brasília, v. 22, N. 2, P.169-174, maio 2006. Disponível em: HTTPS://WWW.RESEARCHGATE.NET/PUBLICATION/262704412_COGNITION_EMOTION_AND_MUSICAL_EXPERTISE. ACESSO EM: 14 SET. 2018.

GALVÃo, Afonso. Estudo deliberado e expertise musical. In: CORRÊA, Antenor Ferreira (Org.). A mente musical em uma perspectiva interdisciPLINAR. Brasília: Universidade de Brasília, 2015. P. 167-189.

KAPlan, José Alberto. Teoria da aprendizagem pianística. 3. ed. Porto Alegre: Movimento, 2008.

MADEIRA, Lígia Raquel Brito. Estratégias de auto-regulação da aprendizagem no ensino Instrumental. 2014. 78 F. DissertaçÃo (Mestrado) Curso de Comunicação e Arte, Universidade de Aveiro - Portugal, Aveiro, 2014. DisponíVEL EM: HTTP://HDL.HANDLE.NET/10773/12925. ACESSO EM: 14 SET. 2018.

MCPHERSON, Gary E.; ZIMMERMAN, Barry J. Self-regulation of music LEARNING: A SOCIAL COGNITIVE PERSPECTIVE ON DEVELOPING PERFORMANCE SKILLS. IN: COLWELL, Richard; WEBSTER, Peter R. MENC HaNdBooK of RESEarch on music learning: Volume 2: Applications. Oxford: OxFord UniVersity Press, 2011. CaP. 4. P. 130-175.

PARIS, Scott G.; PARIS, Alison H. Classroom applications of RESEARCH on Self-regulated learning. Educacional Psychologist, Michigan, n. 362, 
Amostras de ciclos autorregulatórios e sua inserção....

Antenor Ferreira Correa • Luciana Stadniki Martins

P.89-101, 2001. DISPONÍVEL EM: SOHS.PBS.UAM.ES/WEBJESUS/MOTIV_EV_AUTORR/ LeCts\%20extranjeras/autorregulacion.PDF. Acesso em: 14 Set. 2018.

POlydoro, Soely Aparecida Jorge; AZZI, Roberta Gurgel. Auto-regulaÇão: aspectos Introdutórios. In: BANDURA, Albert; AZZI, Roberta Gurgel; POlydoro, Soely. Teoria social cognitiva: conceitos básicos. Porto Alegre: Artmed, 2008. Cap. 7. P. 149-164.

Rosário, Pedro; POlydoro, Soely A. J. Capitanear o aprender: PromoçÃo da Autorregulação da Aprendizagem no Contexto educativo. SÃo Paulo: Casa do Psicólogo, 2014. 3 v. (Teoria Social Cognitiva em Contexto Educativo).

SCHUNK, Dale H.; ZIMMERMAN, Barry J. Influencing Children's Self-Efficacy and Self-Regulation of Reading and Writing Through Modeling. Reading \& Writing Quarterly, v. 23, n. 1, P. 7-25, 2006. Informa UK LIMITED. HTTP://DX.DOI.ORG/10.1080/10573560600837578.

SLOBODA, John A.LA mente musical: LA PSICOlogía COGNITIVA de LA MÚSICA. Madri: Antonio Machado Libros, 2012. Tradução: Beatriz Martín-Andrade e Amalia Casas.

Simão, Ana Maria Veiga. Aprendizagem Estratégica: Uma aposta na aUto-regulação. Lisboa: Ministério da EducaçÃo, 2002. (DesenVolvimento CURRICULAR).

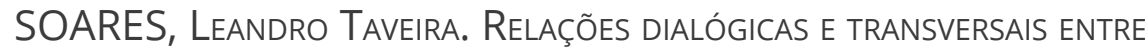
aprendizagem autorregulada e teorias da Aquisição de expertise. Percepta: Revista de Cognição Musical, CuritiBa, v. 2, N. 5, P.93-116, 2018. DispoNÍVEL EM: HTTPS://WWW.ABCOGMUS.ORG/JOURNALS/INDEX.PHP/PERCEPTA/ARTICLE/ VIEW/127. ACESSO EM: 20 JUN. 2019.

WinNe, Philip H; HADWIN, Allyson Fiona. Studying as self-regulated LEARNING. IN: HACKER, DOUgLAS J.; DUNLOSKY, JOHN; GRAESSER, ARTHUR C. Metacognition in educational theory and practice: The educational psychology series. Mahwah: Erlbaum, 1998. Cap.12. p.277-304. DisponíVEL EM: HTTPS://WWW.RESEARCHGATE.NET/PUBLICATION/247664651_STUDYING_ AS_SELF-REGULATED_LEARNING. ACESSO EM: 29 SET. 2018. 
Amostras de ciclos autorregulatórios e sua inserção....

Antenor Ferreira Correa • Luciana Stadniki Martins

ZiMMERMAN, Barry J. Developing Self-Fulfilling cyCles of academic regULATION: AN ANALYSIS OF EXEMPLARY INSTRUCTIONAL MODELS. IN: SCHUNK, DALE H.; ZIMMERMAN, Barry J. (Org.). Self-regulated learning: from teaching to self-reflective practice. New York: Guilford Publications, 1998. CAP. 1, P. 1-19.

Zimmerman, Barry J. Self-efficacy: An essential motive to learn. Contemporary Educational Psychology, v. 25, n. 1, p.82-91, 2000. Elsevier BV. HTTP://DX.DOI.ORG/10.1006/CEPS.1999.1016.

Zimmerman, Barry J. Becoming a Self-Regulated learner: An Overview. Theory Into Practice, Ohio, v. 41, N. 2, P.64-70, jun. 2002. Disponível em: HTTPS://WWW.RESEARCHGATE.NET/PUBLICATION/237065878_BECOMING_A_SELFRegulated_Learner_An_Overview. Acesso em: 28 set. 2018.

ZIMMERMAN, B. J.; CAMPILLO, M. Motivating SElf-Regulated ProBlem solvers. In: DAVIDSON, J. E.; STERNBERG, R. Sternberg (EE.), The nature of problem solving. New York, NY: Cambridge University Press, P. 233-262, 2003. HTTPS://DOI.ORG/10.1017/CBO9780511615771.009.

ZORZAL, Ricieri Carlini. Estratégias para o ensino de instrumento musical. In: ZORZAL, Ricieri CarlinI; TOURINHO, Cristina (Org.). Aspectos prátIcos E TEÓRICOS PARA O ENSINO E APRENDIZAGEM DA PERFORMANCE MUSICAL. SÃO Luís: EdufMA, 2014. CAP.1. P. 12-45.

ZORZAL, Ricieri. Prática musical e planejamento da Performance: CONTRibulÇÕES TEÓRICO-CONCEITUAIS PARA O DESENVOLVIMENTO DA AUTONOMIA DO ESTUDANTE de Instrumento musical. Opus, v. 21, N. 3, P. 83-110, Dez. 2015. OPUS. HTTP://DX.DOI.ORG/10.20504/OPUs2015c2103. 